\title{
Deterministic modeling and evaluation of a virtual research laboratory by using Petri nets
}

\author{
R. P. Romansky \\ Department of Electronics, Computer Systems and Technologies, College of Energy and Electronics, Technical University of Sofia, BULGARIA \\ e-mail: rrom@tu-sofia.bg, Tel +35929653295
}

\begin{abstract}
The purpose of the virtual laboratory is to propose an intellectual space for organization of the on-line work in specialized field of knowledge. The research presented in this paper is linked with the research carried out within the frame work of a two year scientific project. The main goal of the project team is to develop a Virtual Research Laboratory (VRL) as a collection of relatively independent distributed virtual mediums, search machine, software instruments and tools for specific research organization in the field of the modern electronic and computer technologies. The life cycle models for software development propose a system investigation and evaluation by using suitable apparatus to determine the adequate structural organization during the phase of the architectural design. In this reason, the goal of this paper is to present an investigation of the defined architecture by using the asynchronous apparatus of Petri Nets (PN) as a second phase of the project work concerning the VRL development. The PN model defining and the carried out research are based on the results obtained during the first phase of the project work - conceptual model defining, formalization, preliminary evaluation and general architecture building. The formal description is based on the fact that each process of the information servicing in the virtual environment could be treated as a sequence of events (states) that generates requests for access to different information resources (relations) via Internet. This formal description permits to make a deterministic model of the information service as a sequence of active events and transactions between them that could be realized by the asynchronous PN apparatus. The defined PN-model describes the evaluated object as a sequence of events (presented by transactions) that could be realized if all related conditions (presented by input places) are fulfilled. In this reason the PN model describes the information servicing in the evaluated VRL as a collection of transactions and position with defined relations between them. Some important experimental results and assessments are discussed.
\end{abstract}

Keywords: Virtual Laboratory; Architectural Design; System Investigation; Deterministic Modeling; Petri Nets.

\section{Introduction}

Each virtual laboratory is a distributed environment that proposes different possibilities for organization of on-line work, including e-learning and research in a specialized area, for example IT education (Leithner and Cane, 2005), computing and communication technology (Balamuralithara and Woods, 2008), electronic circuit simulation (Ouyang et al, 2005), automatic control (Granado et $a, 2007$ ), mathematics and natural science (Jeschke et al, 2005), etc. For example, a concept of a virtual laboratory that uses the metaphor of scientific lab to emulate scientific hands-on training within a computer network is presented in (Jeschke et al, 2005). In this reason the virtual laboratory should be designed as a bridge between the theoretical knowledge and practical science by using means and tools for experiments realization on the base of computer-implemented algorithms for experiment work simulation in the specialized area of the research. Another approach is discussed in (Way, 2006) - this paper presents an ambitious research laboratory model that permits to build Applied Computing Technology Laboratory (ACT Lab). The students use the shared resources of the laboratory space as own. When they need access to equipment, guidance or information, they succeed in acquiring what they need. The project includes different sections as nanocomputer design, speech recognition, finite automata simulation, measuring, etc. The virtual laboratory model is a structure for a flexible, efficient, and dynamic research program that encourages undergraduate research and collaboration with facilities, other students and industry. 
The building of a virtual environment and including it in the global web world is the goal of the two year scientific project which has started a couple of months ago and should be finished by the end of year 2011. Different resources (e-learning objects, information contents, knowledge, etc.) could be united in a common environment based on distributed technologies and virtual reality so that a shared research could be realized. In this reason, the main goal of the project team is to develop a Virtual Research Laboratory (VRL) as a collection of relatively independent distributed virtual mediums, search machine, software instruments and tools for specific research organization in the field of the modern electronic and computer technologies. A survey of the available virtual applications is made by members of the project team (Radoyska and Spasova, 2010). Another survey on the issue and on digital libraries is published by a member of the project team (Ivanova, 2010), because the main conceptual idea of the virtual environment includes an intelligent search machine and knowledge based library building. The specific problems of the simulation for digital circuits testing and diagnostic for e-learning pourpose are discused in (Radoyska, Ivanova and Spasova, 2010).

The important part of the virtual labs development is the architectural design based on a conceptual model that determines the basic system components and parameters. This model permits to formalize the access processes and information servicing. There are different papers which discuss the problem of architectural design (Afsarmanesh et al, 2010), (Ramasundaram et al, 2005), (Grygárek, Milata and Vavř́ček, 2007). In the last paper a system called Virtlab, which allows to access laboratory equipment remotely via Internet and the architectural development and modifications are discussed. The issues discussed are related to the connectivity, device conflicts, virtual topology, etc. A new distributed architecture based on virtual tunnels is presented and the information security is discussed. In the proposed architecture each site can act as an independent virtual labortory or share its equipment with others. The searching of the devices suitable for distributed virtual topology is dynamic and the architecture incorporates both hardware and software components and is completely based on open-source technologies.

Another important issue discussed is the user interface (UI). A solution for a UI environment developed for the need of virtual laboratory organization is discussed in (Funika et al, 2008). The specialization of the concrete virtual lab and carried out experiments require support in form of a script editor easily extendible by a number of additional functions related to the functionality (sharing experiments, experiment management mechanism, logging errors, etc.). All these components are intended to improve the access to the virtual laboratory resources.

The goal of this paper is to present an investigation of the defined architecture by using the asynchronous apparatus of Petri Nets (PN) as a second phase of the project work concerning the VRL development. The PN model defining and the research is based on the results obtained during the first phase of the project work - conceptual model defining, formalization and preliminary evaluation and general architecture building. To make clear the formulated topic the paper includes different sections: Section $2-$ the problem definition and related work is presented; Section 3 summarizes some details concerning the structural organization, functionality and formalization of the VRL processes and the possibility of the PN modeling; the next sections 4 and 5 discuss the PN model defining, its execution and the evaluation of the basic parameters; a conclusion is made in the final section.

\section{Problem definition and related work}

The general goal of the project is to build an international distributed environment to support research in the field of modern electronic and computer technologies. In this reason the VRL will be realized as a collection of different web-based components united in a common virtual environment. Each component should realize specific functions and will be built as a relatively independent medium in the common distributed environment. In this reason, a conceptual model for the VRL components organization is defined in the first phase of the project. This initial conceptual model determines the main components of the VRL and its purpose. A formalization and stochastic preliminary modeling to prove the functionality and to investigate the relations between information resources and processes of information servicing are made. This modeling is carried out by using the stochastic apparatus of the Markov chains and some of the obtained results and assessments for the probability processes, distributed access and security procedures (authorization and authentication) are discussed in (Romansky, 2010a). The results obtained by this preliminary research help us to determine the general architecture of the designed VRL and to define the purpose of the separate components. The VRL architecture as a collection of the specialized sub-systems, digital knowledge library and intelligent searching machine is presented in (Romansky, 2010b). Additional functional modeling by using Data Flow Diagram (DFD-model) and UML-model (Activities Diagram) is presented in the same paper.

The life cycle models for software development propose to make a system investigation and evaluation by using suitable apparatus to determine the adequate structural organization during the phase of the architectural design. This problem is resolved in (Liegle and Meso, 2007) as a case study to evaluate the aspects of the virtual computing to the effectiveness of the teaching distributed applications development. The paper explores how the virtual lab effectively addresses many of the challenges of teaching application development. A case study is provided and the results are obtained by survey on students' experience and perception of the virtual lab. A workflow models to summarize the steps for the $3 \mathrm{D}$ profile creating is used in the virtual lab in (Ramasundaram et al, 2005). Different means of assessing virtual reality performance have been described in (Seymour, 2008), including motion analysis, hidden Markov models, simulation, etc. All these means are used for testing and validation of the performed processes.

The object of this paper is to discuss an investigation of the presented in (Romansky, 2010b) VRL architecture. It will be better if different means for investigation are used in the separate phases of the VRL building. In this reason, the goal of this 
investigation is defined as a secondary evaluation of the information servicing and communications between VRL components. Because the processes in a distributed structure are situated in different nodes a graph description for the formalization assuming discrete states and deterministic nature of the transactions is made. The reason for this is the fact that each process of the information servicing in the virtual environment could be regarded as a sequence of events (states) that generates requests for access to different information resources (relations) via Internet. We assume that each user $U_{i}$ realizes a remote access to resources in different nodes by request directed trough the transmitter $T_{q}$ by the assistance of any distributor $D$. The abstract model is presented in Figure 1. The resources present information objects, knowledge, contents, tools, etc, that permit multi-user access. The transmitters present technical and technological tools in the global network for communications and the distributors describe the tools for routing all requests and results between sources and destinations. This abstraction permits to formalize the virtual environment as an ordered discrete structure $\{U, R, D, T\}$ with two types of relations request: $U_{i} \stackrel{T q}{\longrightarrow} R_{j}$ and result : $R_{j} \stackrel{T q}{\longrightarrow} D_{i}$.

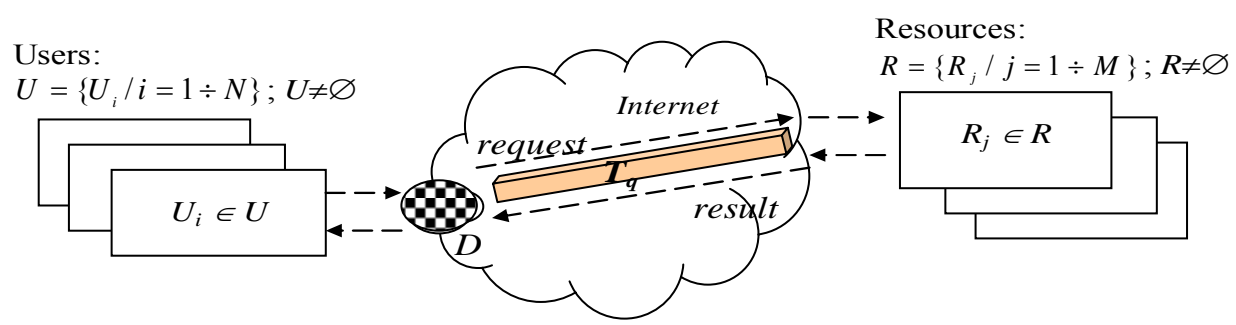

Figure 1. Abstract model of the remote access to the resources

The PN apparatus could be used to describe the asynchronous processes in this virtual environment because it is a discrete mathematical structure with a big power for an investigation of the information servicing and remote access to the distributed VRL components. Another argument is that the results from the PN modeling will propose to verify the assessment obtained by the preliminary stochastic investigation.

\section{Structural organization and formal description of the information processes}

The main structural organization of the developed VRL is shown in Figure 2. This structure generalizes the architecture presented in (Romansky, 2010b) as a collection of two sub-systems: front-office and back-office.

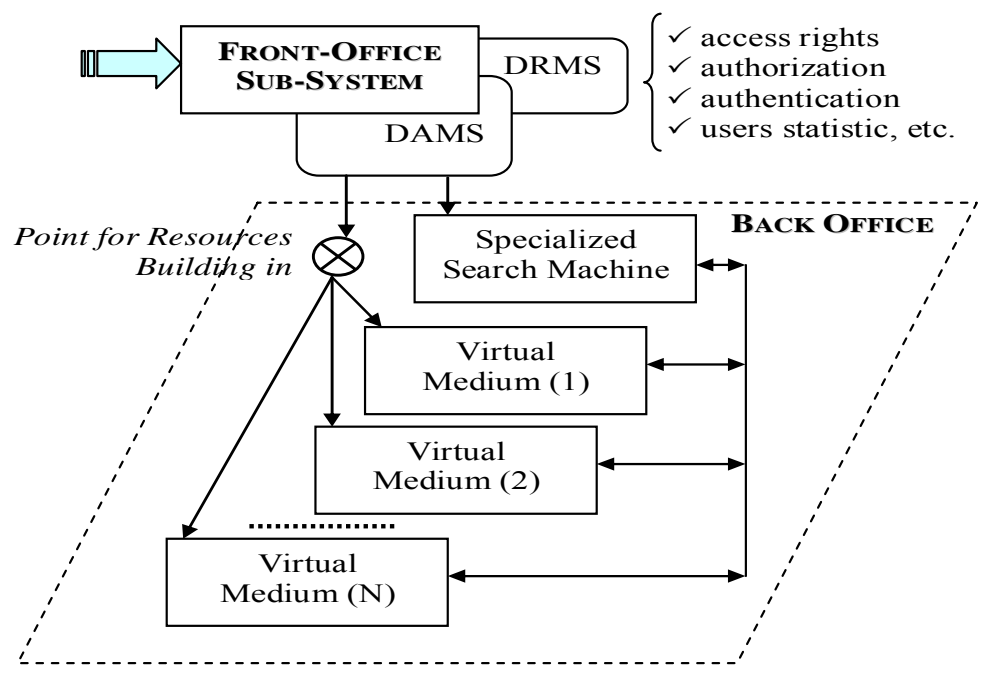

Figure 2. The main structural organization of the VRL

1. Front-office system as a web-based interface for communication between users and virtual components. Two very important components should be developed to manage the distributed access to the VRL resources:

$\checkmark$ Digital Rights Management System (DRMS) - to support the high level of information security and to manage the access to the resource by digital rights checking, authorization, authentication, and collect of the users' statistic; 
$\checkmark$ Distributed Access Management Systems (DAMS) - for managing the user's access to the resources (internal and external). The basic tasks of DAMS include administrative work such as user and resources management, contents and knowledge submissions, e-mail notification of announcement, etc.

2. Back-office system as a collection of different virtual mediums and instruments for research supporting and intelligent searching. This sub-system should give the following components and services:

$\checkmark$ medium for exchange of knowledge and ideas realized by web-based two-language interactive multimedia design and elearning technologies;

$\checkmark$ dynamic management of access and resources;

$\checkmark$ specialized search machine;

$\checkmark$ e-space and instruments for research results publishing;

$\checkmark$ medium for virtual presentations and e-meetings;

$\checkmark$ separate virtual mediums for carrying out research experiments in different fields and analysis of the results;

$\checkmark$ a possibility for building in new virtual mediums to extend the purpose of the VRL.

A formalization of this functionality could be made by using the states transition diagram shown in figure 3 . The main functional processes in the VRL are described as separate states of the diagram and the relations are presented (by the transaction).

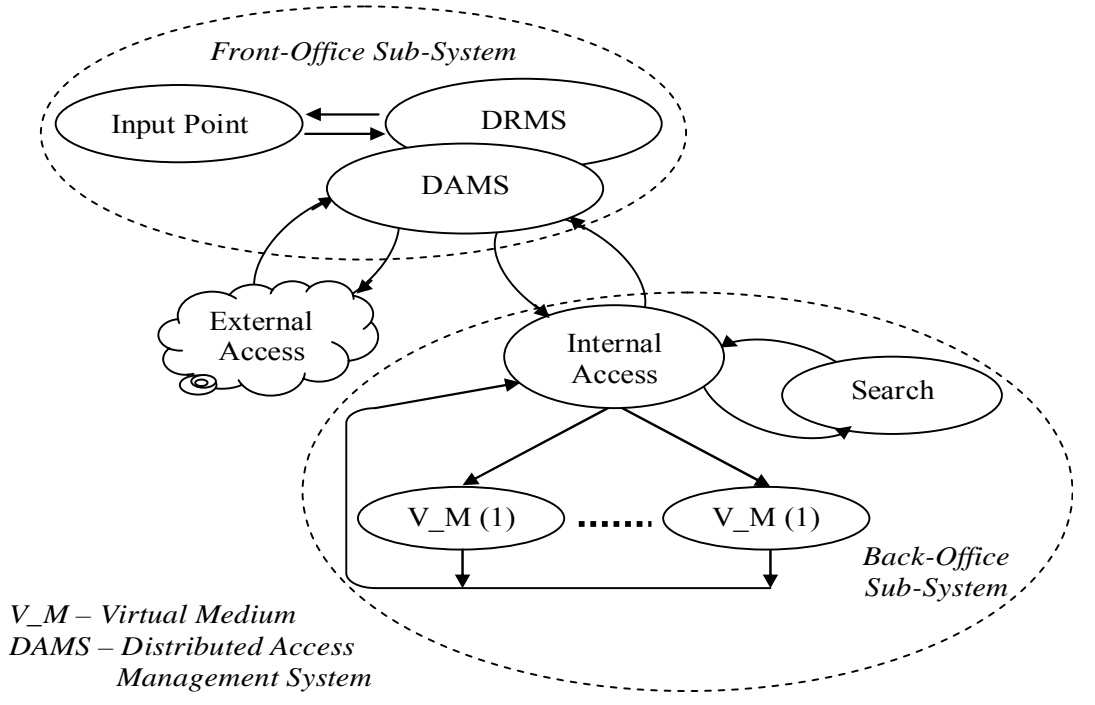

Figure 3. Formalization of the information processes in the VRL

This formal description permits to make a deterministic model of the information service as a sequence of active events and transactions between them that could be realized by the asynchronous PN apparatus. A PN-model could describe the evaluated object as a sequence of events (presented by transactions) that could be realized if all related conditions (presented by input places) are fulfilled. In this reason the PN model describes the information servicing in the evaluated VRL as a collection of transactions and position with defined relations between them.

The classic PN model could be defined by the ordered structure $P N=\left(P, T, F, W, \mu_{0}\right)$ based on the set of the places (conditions) $P=\left\{p_{1}, p_{2}, \ldots, p_{\mathrm{m}}\right\}$, the set of the transactions (events) $T=\left\{t_{1}, t_{2}, \ldots, t_{\mathrm{n}}\right\}$, the set of relations (input and output functions) $F \subseteq(P \times T) \cup(T \times P)$, the weight function of the relations $W: F \rightarrow\{1,2,3, \ldots\}$ and the initial marking $\mu_{0}: P \rightarrow\{0,1,2,3, \ldots\}$ for the beginning of the model execution. The sets $P$ and $T$ are final sets $(m \geq 0, n \geq 0, P \cap T=\varnothing)$. The set $F$ includes ordered couples $\left(p_{\mathrm{i}}\right.$, $t_{\mathrm{j}}$ ) that define relations $P \rightarrow T$ and $T \rightarrow P$, and two functions are constructed $-I, O \in \mathbf{N}^{|\mathrm{P} \times| \mathrm{T} \mid}$, named input and output functions. The PN-model could be graphically presented by using of directed multi-graph with the states the elements of the sets ra $P$ and $T$, and transactions defined by the functions $I \in F$ (input function) and $O \in F$ (output function). Each transaction $\left(t_{\mathrm{j}}\right)$ can have one or more input places (defined by the function $I$ to present the conditions which activate this event), and some output places (defined by function $O$ ) which relate to the next event(s) that could be activated during the model execution.

An additional description of the PN-model could be given by the input $\left(\mathbf{D}^{-}\right)$and output $\left(\mathbf{D}^{+}\right)$matrixes, defined by the following formalism: $P N=\left(P, T, \mathbf{D}^{-}, \mathbf{D}^{+}\right) ; \mathbf{D}^{-}[j, i]=\eta\left(p_{i}, I\left(t_{j}\right)\right) ; \forall p_{i} \in P ; j=1 \div|T| ; \mathbf{D}^{+}[j, i]=\eta\left(p_{i}, O\left(t_{j}\right)\right) ; \forall p_{i} \in P ; j=1 \div|T|$. This definition permits each transaction $t_{j}$ to be presented by a vector $e[j](m=|T|)$ consists of $m$ binary digits with " 1 " in the position,,$j$ " only.

The modeling by using PN is based on execution of marked net that each position has an integer count of marks $k \geq 0$ that could be changed during the PN evolution $\mu_{0} \rightarrow \mu_{1} \rightarrow \mu_{2} \rightarrow \ldots$. 


\section{Definition of the PN-model}

The definition of the PN-model is based on the following events (presented by transactions) and the conditions for the activation of the events (presented by places):

$t_{1}$ - user registration;

$t_{2}$ - system administrator registration;

$t_{3}$ - authentication (identification);

$t_{4}$ - authorization (digital right checking);

$t_{5}$ - input into the back office system;

$t_{6}$ - output (finishing of the work with the VRL);

$t_{7}$ - search machine using;

$t_{8}$ - application level of work with the VRL resources;

$t_{9}$ - system level of work with the VRL resources.

The theoretical definition of the model is presented below:

$P=\left\{p_{1}, p_{2}, p_{3}, p_{4}, p_{5}, p_{6}\right\}$

$T=\left\{t_{1}, t_{2}, t_{3}, t_{4}, t_{5}, t_{6}, t_{7}, t_{8}, t_{9}\right\}$
$I\left(t_{1}\right)=\left\{p_{1}, p_{1}\right\}$

$I\left(t_{2}\right)=\left\{p_{1}, p_{1}\right\}$

$I\left(t_{3}\right)=\left\{p_{1}, p_{2}\right\}$

$I\left(t_{4}\right)=\left\{p_{3}\right\}$

$I\left(t_{5}\right)=\left\{p_{3}, p_{4}\right\}$

$I\left(t_{6}\right)=\left\{p_{5}\right\}$

$I\left(t_{7}\right)=\left\{p_{5}\right\}$

$I\left(t_{8}\right)=\left\{p_{5}\right\}$

$I\left(t_{9}\right)=\left\{p_{5}, p_{6}, p_{6}, p_{6}\right\}$ $p_{1}-$ available request for access to the front-office system;

$p_{2}$ - correct registered user;

$p_{3}-$ successful identification;

$p_{4}$ - successful authorization;

$p_{5}-$ available request for servicing;

$p_{6}-$ permitted high level access (system level).

The two matrixes (input and output) are defined below:

\begin{tabular}{c|c|c|c|c|c|c|}
$\mathbf{D}^{-}$ & \multicolumn{1}{c}{$p_{1}$} & $p_{2}$ & $p_{3}$ & $p_{4}$ & $p_{5}$ & $p_{6}$ \\
\cline { 2 - 7 }$t_{1}$ & $\mathbf{2}$ & 0 & 0 & 0 & 0 & 0 \\
\cline { 2 - 7 }$t_{2}$ & $\mathbf{2}$ & 0 & 0 & 0 & 0 & 0 \\
\cline { 2 - 7 }$t_{3}$ & $\mathbf{1}$ & $\mathbf{1}$ & 0 & 0 & 0 & 0 \\
\cline { 2 - 7 }$t_{4}$ & 0 & 0 & $\mathbf{1}$ & 0 & 0 & 0 \\
\cline { 2 - 7 }$t_{5}$ & 0 & 0 & $\mathbf{1}$ & $\mathbf{1}$ & 0 & 0 \\
\cline { 2 - 7 }$t_{6}$ & 0 & 0 & 0 & 0 & $\mathbf{1}$ & 0 \\
$t_{7}$ & 0 & 0 & 0 & 0 & $\mathbf{1}$ & 0 \\
\cline { 2 - 7 }$t_{8}$ & 0 & 0 & 0 & 0 & $\mathbf{1}$ & 0 \\
\hline$t_{9}$ & 0 & 0 & 0 & 0 & $\mathbf{1}$ & $\mathbf{3}$ \\
\hline
\end{tabular}

\begin{tabular}{c|c|c|c|c|c|c|}
$\mathbf{D}^{+}$ & \multicolumn{1}{c}{$p_{1}$} & \multicolumn{1}{c}{$p_{2}$} & $p_{3}$ & $p_{4}$ & $p_{5}$ & $p_{6}$ \\
\cline { 2 - 7 }$t_{1}$ & $\mathbf{1}$ & $\mathbf{1}$ & 0 & 0 & 0 & 0 \\
\cline { 2 - 7 }$t_{2}$ & $\mathbf{1}$ & $\mathbf{1}$ & 0 & 0 & 0 & $\mathbf{1}$ \\
\cline { 2 - 7 }$t_{3}$ & 0 & 0 & $\mathbf{2}$ & 0 & 0 & $\mathbf{1}$ \\
\cline { 2 - 7 }$t_{4}$ & 0 & 0 & 0 & $\mathbf{1}$ & 0 & $\mathbf{1}$ \\
\cline { 2 - 7 }$t_{5}$ & 0 & 0 & 0 & 0 & $\mathbf{1}$ & 0 \\
\cline { 2 - 7 }$t_{6}$ & 0 & 0 & 0 & 0 & 0 & 0 \\
$t_{7}$ & 0 & 0 & 0 & 0 & $\mathbf{1}$ & 0 \\
\cline { 2 - 7 }$t_{8}$ & 0 & 0 & 0 & 0 & $\mathbf{1}$ & 0 \\
\cline { 2 - 7 }$t_{9}$ & 0 & 0 & 0 & 0 & $\mathbf{1}$ & $\mathbf{3}$ \\
\hline
\end{tabular}

The multi-graph of the defined PN-model (graph presentation) is shown in figure 4.

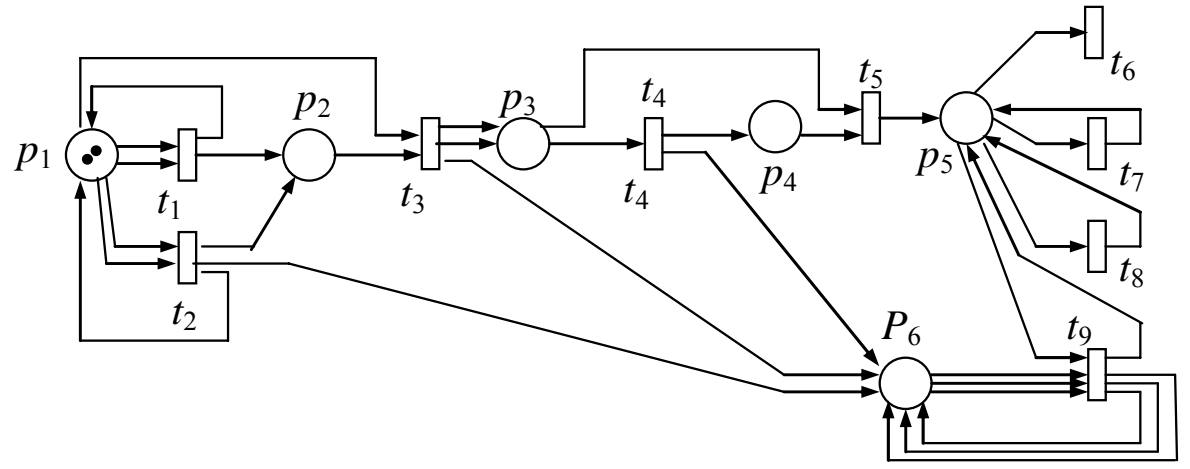

Figure 4. Graph presentation of the model with an initial marking $\mu_{0}=(2,0,0,0,0,0)$ 


\section{Model execution and parameters' evaluation}

The execution of the defined model could be carried out based on the current marking started from the initial marking $\mu_{0}$. Each marking is a numeric vector of $n$ elements ( $n$ is the number of the places in the set $P$ ) $\mu=\left(\sigma_{1}, \sigma_{2}, \ldots, \sigma_{n}\right)$ that the digit $n_{i}$ shows the current count of marks in the place $p_{i}$. A transaction $t_{j}$ could by activated if all relevant conditions determined by $I\left(t_{\mathrm{j}}\right)$ are fulfilled. If a transaction is activated some marks are transferred trough this transaction to the output places according the corresponding function $O\left(t_{\mathrm{j}}\right)$. The model execution is an evolution of the PN - it is formed the chain of markings $\mu_{0} \rightarrow \mu_{1} \rightarrow \mu_{2} \rightarrow \ldots$ based on the permitted markings. In this reason the first task is to define the permitted transaction(s) for the initial marking and for each of the steps of the model execution. If the matrix approach is used the condition $\mu \geq e[j] . D^{-}$should be checked for the each transaction $t_{j}$. The next marking $\mu^{*}$ (if the transaction $t_{j}$ is activated) could be determined on the base of the equation $\mu^{*}=\mu+e[j] . D$, where $D=D^{+}-D^{-}$is called incident matrix. The evolution of the model could be presented by the tree of the reachability that could be constructed based on all possible chains of markings.

\section{- Determination of the permitted transactions}

The condition $\mu \geq e[j] . D^{-}$should be checked for each transaction to determine which of them could be activated at the initial marking $\mu_{0}$ :

$$
\begin{aligned}
& t_{1} \quad \Rightarrow \quad \mu_{0}=(2,0,0,0,0,0) \geq(1,0,0,0,0,0,0,0,0) \cdot D^{-}=(2,0,0,0,0,0) \quad \Rightarrow \quad \text { Yes } \\
& t_{2} \quad \Rightarrow \quad \mu_{0}=(2,0,0,0,0,0) \geq(0,1,0,0,0,0,0,0,0) \cdot D^{-}=(2,0,0,0,0,0) \quad \Rightarrow \quad \text { Yes } \\
& t_{3} \quad \Rightarrow \quad \mu_{0}=(2,0,0,0,0,0) \geq(0,0,1,0,0,0,0,0,0) \cdot D^{-}=(1,1,0,0,0,0) \quad \Rightarrow \quad \text { No } \\
& t_{4} \quad \Rightarrow \quad \mu_{0}=(2,0,0,0,0,0) \geq(0,0,0,1,0,0,0,0,0) \cdot D^{-}=(0,0,1,0,0,0) \quad \Rightarrow \quad \text { No } \\
& t_{5} \quad \Rightarrow \quad \mu_{0}=(2,0,0,0,0,0) \geq(0,0,0,0,1,0,0,0,0) \cdot D^{-}=(0,0,1,1,0,0) \quad \Rightarrow \quad \text { No } \\
& t_{6} \quad \Rightarrow \quad \mu_{0}=(2,0,0,0,0,0) \geq(0,0,0,0,0,0,1,0,0) \cdot D^{-}=(0,0,0,0,1,0) \\
& t_{7} \quad \Rightarrow \quad \mu_{0}=(2,0,0,0,0,0) \geq(0,0,0,0,0,0,1,0,0) \cdot D^{-}=(0,0,0,0,1,0) \quad \Rightarrow \text { No } \\
& t_{8} \quad \Rightarrow \quad \mu_{0}=(2,0,0,0,0,0) \geq(0,0,0,0,0,0,0,1,0) \cdot D^{-}=(0,0,0,0,1,0) \quad \Rightarrow \quad \text { No } \\
& t_{9} \quad \Rightarrow \quad \mu_{0}=(2,0,0,0,0,0) \geq(0,0,0,0,0,0,0,0,1) \cdot D^{-}=(0,0,0,0,1,3) \quad \Rightarrow \text { No }
\end{aligned}
$$

The result shows that only the transactions $t_{1}$ and $t_{2}$ are permitted and could be activated because their conditions are fulfilled.

\section{- Determination of the next markings}

The equation $\mu_{i+1}=\mu_{i}+e[j] . D$ should be calculated to find the solution of this task. The incident matrix is calculated as a first step:

$$
D=D^{+}-D^{-}=\left[\begin{array}{rrrrrr}
-1,-1, & 0, & 0, & 0, & 0 \\
-1,+1, & 0, & 0, & 0, & +1 \\
-1, & -1, & +2, & 0, & 0, & +1 \\
0, & 0, & -1, & +1, & 0, & +1 \\
0, & 0, & -1, & -1, & +1, & 0 \\
0, & 0, & 0, & 0, & -1, & 0 \\
0, & 0, & 0, & 0, & 0, & 0 \\
0, & 0, & 0, & 0, & 0, & 0 \\
0, & 0, & 0, & 0, & 0, & 0
\end{array}\right]
$$

The solutions for the two permitted transactions are as follows:

$\checkmark$ for $t_{1}: \quad \mu_{\mathrm{t} 1}=(2,0,0,0,0,0)+(1,0,0,0,0,0,0,0,0) \cdot D=(2,0,0,0,0,0)+(-1,1,0,0,0,0)=(1,1,0,0,0,0)$;

$\checkmark$ for $t_{2}: \quad \mu_{\mathrm{t} 2}=(2,0,0,0,0,0)+(0,1,0,0,0,0,0,0,0) \cdot D=(2,0,0,0,0,0)+(-1,1,0,0,0,1)=(1,1,0,0,0,1)$.

They correspond to the two relatively independent branches of work with the resources of the VRL (Figure 5), determined by the digital rights management system. 


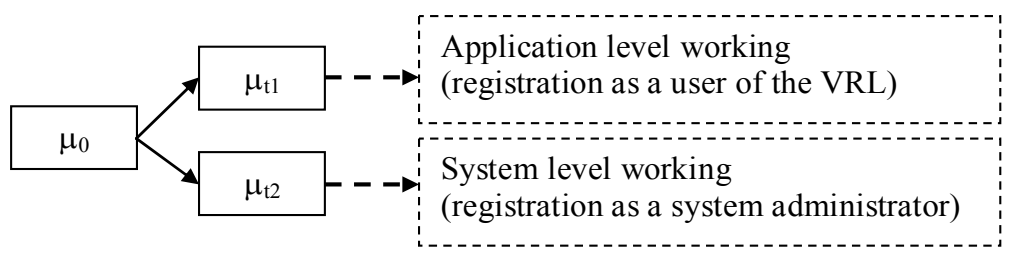

Figure 5. Two branches of the work with the VRL resources

\section{- Determination of the tree of reachability}

The tree of reachablity determined by realization the upper steps for all possible transaction is shown in figure 6 .

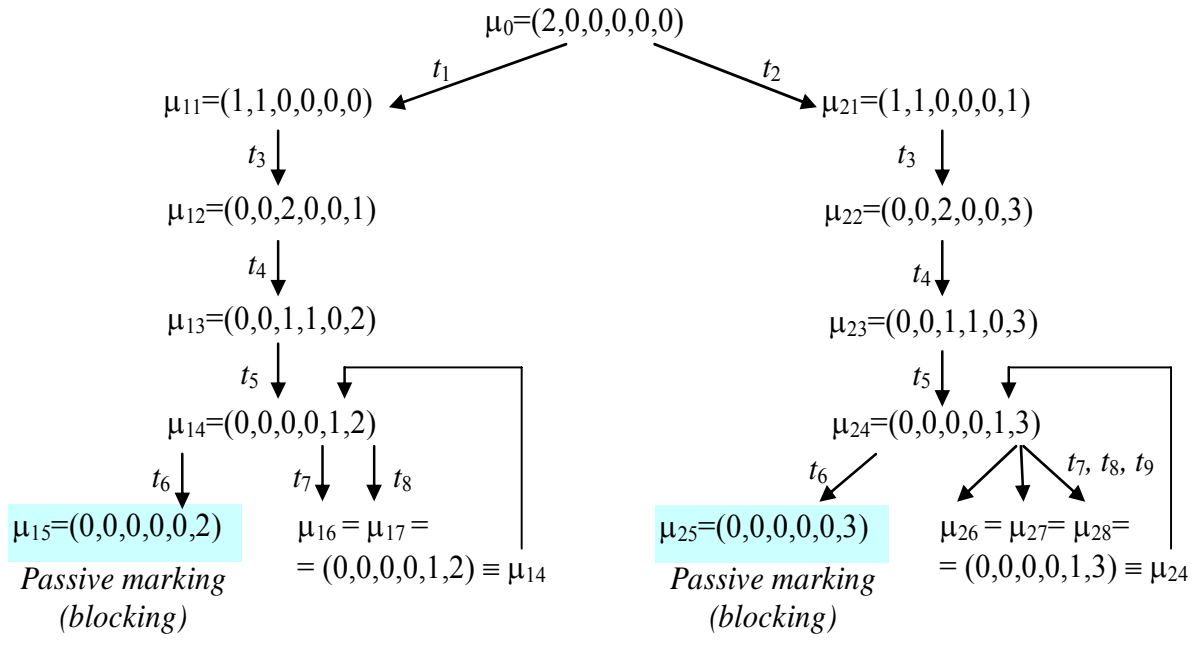

Figure 6. Tree of reachability for the PN-model

The evolution (represented by the tree from the figure 6) defines the execution of the model as a sequence of states determined by chain of activated events. Each event could be realized at a given moment if the corresponding conditions are performed. The model execution could be described as a scheme of evolution, shown in figure 7. Each block of this scheme consists of some numbers that give information about the available marks in the places. For example, the information " $3,3,6$ " defines 2 marks in the place $p_{3}$ and 1 mark in the place $p_{6}$.

Two levels are formed to present the profile of the current active user - both as a user and as a system administrator. Each of the branches finishes with the sector responsible to work with the back-office system (activation of the transactions $t_{7}, t_{8}$ or $t_{9}$ ) or end of work with the VRL (activation of the transaction $t_{6}$ and output). The point "END" in the scheme shows that the process has finished and a passive marking is formed after the transaction $t_{6}$ is activated. This problem exists because the scheme of the evolution presents only one phase execution of the model. To ignore this problem it is possible to add two arcs between the transaction $t_{6}$ (for each branch) and the transaction $t_{1}$ - in this case the model will go to the state "readiness" (initial marking $\mu_{0}$ ).

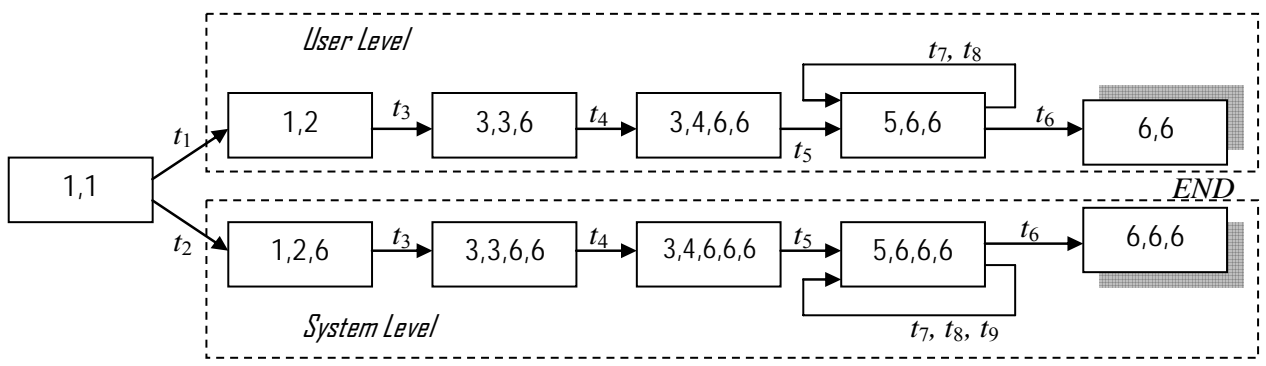

Figure 7. Scheme of the model evolution 


\section{- Analysis of the defined model}

The model investigation is realized by the analysis of the tree of the reachability and of the basic model conditions. The main results are generalized below.

$\checkmark$ Reachability - each transaction could be reached at concrete situation and this determines the accessibility of all components (resources) of the virtual environment based on user's requests;

$\checkmark$ Liveliness -it could be accepted that the model is alive because during the execution (beginning from the initial marking) for each step exists at least one permitted transaction (for the transaction $t_{6}$ this characteristic will be valid only if we add two arcs);

$\checkmark$ Blocking - the model has a blocked situation only at the one-way version of execution (single use of the VRL). The model in the multi-use version has not blockages and it is active;

$\checkmark$ Boundness - the model is 3-bounded because $\Sigma \mu\left(p_{i}\right) \leq 3$ (see Figure 6);

$\checkmark$ Safety - it may be accepted that the model is safe because for all transactions the number of input arcs is equal to the number of output arcs.

$\checkmark$ Persistence and Conservativeness - these attributes are not available because the total number of marks is not constant.

\section{Conclusions and future work}

The paper deals with some problems of the architectural design of distributed environment to support research as an international virtual laboratory. It is known that the software system should be developed following suitable life cycle model. The article discuses one of the important phases in the each life cycle model - evaluation of the system architecture of the designed object (in this case distributed environment) before the real program realization.

The main goal of this paper is to present the organization of the modeling and system characteristics evaluation as a step in the phase of the architectural design. This investigation (based on defined model) is very important to detect hidden defects in the preliminary defined structure and communications between components. That's why the approach of deterministic modeling for describing the architecture of the virtual environment is used. The using of the asynchronous apparatus of Petri nets permits us to obtain results for the resources accessibility and evaluate some characteristics of the developed environment.

The results obtained by this investigation should be regarded in the context of previously and future phases, realized in the frame of the current research project that will be finished in the end of the year 2011. In this reason the carried out modeling and system evaluation will be used as a basis for detailed architectural design and structure organization of VRL as a multi-component international environment included in the global web-space. Preliminary conversations about this are carried out with our colleagues from educational and research institutions form Slovakia and Greece.

\section{Acknowledgement}

The results in this paper are supported by the Project 102ni013-10 of the Research \& Developing Sector of the Technical University of Sofia, Bulgaria.

\section{References}

Afsarmanesh H., A. Benabdelkader, E.C. Kaletas, C. Garita, L.O. Hertzberger, 2010. Towards a multi-layer architecture for scientific virtual laboratories. In book High Performance Computing and Network, pp. 163-176.

Balamuralithara B., P.C. Woods, 2008. Virtual laboratories in engineering education: The simulation lab and remote lab. Computer Applications in Engineering Education, Vol.17, No 1, pp.101-118.

Funika W., D. Harezlak, D. Krol, M. Bubak, 2008. Environment for collaborative development and execution of virtual laboratory applications. Lecture Notes on Computer Science, Vol.5103, Springer-Verlag Berlin, pp.446-455.

Granado E., W. Colmenares, M. Strefezza, A. Alonso, 2007. A web-based virtual laboratory for teaching automatic control. Computer Applications in Engineering Education, Vol.15, No 2, pp. 192-197.

Grygárek P., M. Milata, J. Vavříček, 2007. The fully distributed architecture of virtual network laboratory, $5^{\text {th }}$ Int. Conference on Emerging e-learning Technologies and Applications, The High Tatras, Slovakia, 6-8 September, pp.1-6.

Ivanova T., 2010. Searching Scientific Digital Libraries - Survey and Modern Trends. Proceedings of the International Conference on Information Technologies (InfoTech-2010), ISSN 1314-1023, 16-17 September, St. Constantine and Elena, Bulgaria, pp.305-312.

Jeschke S., T. Richter, R. Seiler, 2005. VideoEasel: architecture of virtual laboratories for mathematics and natural sciences, $3^{\text {rd }}$ Int'l Conf. on Multimedia and Information and Communication Technology in Education, 7-10 June (in book Recent Research Developments in Learning Technologies), http://www.formatex.org/micte2005.

Leithner L, W. Cane, 2005. A virtual laboratory environment for online it education, Proceedings of the $6^{\text {th }}$ Conference on IT Education, USA (ISBN 1-59593-252-6), pp.283-289. 
Liegle J., P. Meso, 2007. Evaluation of a virtual lab environment for teaching web application development. Information Systems Education Journal, Vol.5, No 7, pp.3-11.

Ouyang Yang, Dong Yabo, Zhu Miaoliang, Huang Yuewei, Mao Song, Mao Yunjie, 2005. ECVlab: A web-based virtual laboratory system for electronic circuit simulation. Lecture Notes in Computer Science, Vol. 3514, pp.1027-1034.

Radoyska P., T. Ivanova, N. Spasova, 2010. Simulation e-Learning environment for digital circuits test and diagnosis, Conference ICL2010. ISBN 98-3-89958-541-4, 15-17 September, Hasselt, Belgium, pp.1043-1050.

Radoyska P., N. Spasova, 2010. Virtual research laboratories in the field of electronic and computer technologies. Proceedings of the International Conference on Information Technologies (InfoTech-2010). ISSN 1314-1023, 16-17 September, St. Constantine and Elena, Bulgaria, pp.263-270.

Ramasundaram V., S. Grunwald, A. Mangeot, N.B. Comerford, C.M. Bliss, 2005. Development of an environmental virtual field laboratory. Computer and Education, No 45, pp.21-34.

Romansky R., 2010a. Virtual research laboratory - a conceptual model and preliminary stochastic investigation, Proceedings of the $6^{\text {th }}$ Int'l Conference on e-Learning and Knowledge Society (e-Learning'10), ISBN 978-5-9984-30-181-5, 26-27 August, Riga, Latvia, pp.136-141.

Romansky R., 2010b. Functional modeling and investigation of a virtual environment for research. Proceedings of the $10^{\text {th }}$ International Scientific Conference UNITECH'10, Vol.I (ISSN 1313-230X), 19-20 November, Gabrovo, Bulgaria, pp.418-423.

Seymour N. E., 2008. VR to OR: A review of the evidence that virtual reality simulation improves operating room performance. World Journal of Surgery, Vol.32, No 2, pp.182-188.

Way T., 2006. A virtual laboratory model for encouraging undergraduate research. ACM Conference SIGCSE, 1-5 March, Huston, Texas, USA.

\section{Biographical notes}

R. P. Romansky received M.Sc. in Computer Engineering (CE), M.Sc. in Applied Mathematics (AM) and Ph.D. in CE from Technical University of Sofia, Bulgaria in 1979, 1980 and 1987, respectively; Associated Professor (since 1996) and Full Professor on Computer Systems, Complexes and Networks (since 2010). Presently hi is a Head of Department on Electronics, Computer Systems and Technologies, College of Energy and Electronics at Technical University of Sofia. His research interests include computer systems and information technology, computer architectures, parallel and distributed systems, computer modeling, data protection. He has over 160 scientific publications and 16 published books. He is a member of the Bulgarian Union of Scientists, Union of Electronics, Electrical Engineers and Communication, Union of Automatics and Informatics and an affiliate member of the European Network of Excellence on High Performance and Embedded Architectures and Compilation.

Received September 2010

Accepted December 2010

Final acceptance in revised form December 2010 